\title{
Die Verbindung des Vorderhirns mit dem metameren Hirn.
}

\author{
Von \\ B. Haller. \\ Hierzu Tafel XXII und 1 Textfigur.
}

Der erste, der die Grosshirn- oder Vorderhirnbahnen der Fische verfolgt hatte, war bekanntlich $1888 \mathrm{Edinger}$ (2). In einer zweiten Schrift (3) gab er dann drei Jahre später Ergänzungen zu seinen früheren Befunden.

Es verbindet sich nach ihm das Vorderhirn durch mehrere Bahnen mit dem $Z$ wischenhirn, nämlich durch das basale Vorderhirnbündel, das Mantelbündel und das Habenularbündel. Das basale Vorderhirnbündel entspringt bei den Knochenfischen mit drei Wurzeln im Vorderhirn, und zwar mit zweien aus dem dorsalen Vorderhirngebiet, mit einer dritten aus dem medianen. Bei den Selachiern aus dem Stammganglion entspringend, gelangt das basale Vorderhirnbündel basalwärts und da die Chiasma optica und die Commissura postoptica überschreitend, begibt es sich in das Zwischenhirn. Zum Teil endet es da im Thalamusgebiet, ein Bündel davon zieht aber weiter kaudalwärts und konnte bis in die Oblongata verfolgt werden.

Eine teilweise Kreuzung der Bündel der basalen Vorderhirnbahn erfolgt in der Commissura postoptica.

Aus der dorsalen Mantelregion entspringend, zieht die jederseitige Mantelbahn ziemlich senkrecht am hinteren Vorderhirnrande hinab zur Basis hinter das Chiasma, dort kreuzen sich die beiderseitigen Bündel in der Commissura postoptica, um dann von hier aus kaudalwärts zu ziehen. Heute ist es festgestellt, dass sie im thalamalen Gebiete verbleiben.

Die Habenularbahn verbindet das Vorderhirn mit dem Habenularganglion.

1898 habe ich im ersten Teil (7) meines Hirnwerkes auch über das basale Vorderhirnbündel bei Fischen berichtet und mitgeteilt, dass es, wie $\mathbf{E}$ ding e $\mathbf{r}$ angab, entspringend und verlaufend innerhalb des Lobus inferior in meinem Vereinsgebiete endet. Eine 
Kreuzung von Vorderhirnfasern in der Commissura anterior geht diese Bahn nichts an, aber es gelangt von ihr aus auch zu keiner Kreuzung in der Commissura postoptica. Bestritten hatte ich eine Verlängerung in das metamere Hirn aber auch. An dem Mantelbündel unterschied ich einen dorsalen und einen ventralen $A b$ schnitt; ersterer ist die Habenularbahn. Der ventrale kommt peripher ventralst von der Opticuswurzel gelegen zur Commissura postoptica, sich dort kreuzend, aber ein Teil bleibt ungekreuzt. Dieses gesamte untere Mantelbündel endet im Vereinsgebiet des Zwischenhirns und gelangt - wie ich dies auch jetzt vertrete - nicht in das metamere Gehirn.

Inzwischen hat sich die Sache dahin gestaltet, dass bezüglich des basalen Vorderhirnbündels meine Angaben, wonach aus diesen kein Nebenbündel in das metamere Gehirn gerät (Edinge r, 1888) allgemein angenommen und das ganze Bündel infolgedessen den Namen Tractus strio-thalamicus erhalten bat. Auch bezüglich des Mantelbündels wird angegeben - ich führe nur Edinger $(4,5)$, Kappers (12) und Goldstein (6) an - dass nach der Kreuzung in der Commissura postoptica die Fasern im Vereinsgebiet verbleiben. Bezüglich des Mantelbündels bin ich auch jetzt derselben Meinung und Edingers Irrtum wäre somit beseitigt. Gleichzeitig möchte ich aber einen Irrtum auch meinerseits feststellen bezüglich des basalen Vorderhirnbündels, denn Edinger hatte 1888 recht, als er eine Verlängerung eines Nebenbündels vom Hauptbündel in das metamere Hirn (er sagt in die Oblongata) behauptete. Er bestand aber damals nicht fest genug auf seinem Befund und heute hat er ihn fallen lassen. Der einzige ist, soviel mir bekannt, Friedr. Mayer in Prag (14), der darauf besteht, dass bei Ammocoetes Fasern aus dem Vorderhirn direkt, also ohne Unterbrechung, allerdings gekreuzt in der Commissura anterior, was unrichtig ist, Fasern in das metamere Gehirn geraten.

Ähnlich steht es bezüglich der Reptilien. Da war es mir auch nicht gelungen (8), einen direkten Übergang des basalen Vorderhirnbündels in das metamere Hirn festzustellen, denn wie es sich weiter unten zeigen wird, habe ich am unrichtigen Orte darnach gesucht. So scheint es auch Unger (15) sieben Jahre später ergangen zu sein. Ich liess alle Fasern des basalen Vorderhirnbündels nur vermittelst des Vereinsgebietes mit dem 
metameren Gehirn in Verbindung treten. Dies schien mir um so sicherer zu sein, als es mir gelungen war, eine Endigung der Pyramidenbahn im Vereinsgebiet, speziell im Lobus inferior festzustellen.

Bei den Să ugeti e r en besteht das basale Vorderhirnbündel, das auch als Grosshirnschenkel bezeichnet wird, aus vier verschiedenen Bahnen: 1. dem Tractus cortico-spinalis, 2. dem Tractus cortico-pontinus, 3. dem Tractus cortico-bulbaris und 4. dem Tractus strio-thalamicus.

Die drei ersteren werden den Fischen gegenüber von Edinger (5) als Neubildungen erklärt. Denn er nimmt heute an, dass die Grosshirnrindenbahnen, „erst relativ spät in der Reihe auftreten und noch viel später erst eine gewisse Vollkommenheit erreichen; ja, dass solche Bahnen erst bei den Säugern zu der Mehrzahl der anderen Hirnteile in Beziehung treten", $(4$, S. 242$)$.

Der Tractus cortico-spinalis entsteht in der Grosshirnrinde aus der vorderen Zentralwindung bei dem Menschen und wird zur Pyramidenbahn. Der Tractus cortico-pontinus stammt aus dem Stirnlappen, dann aus dem Temporal- und Occipitallappen und soll in der Brücke enden. Der Tractus cortico-bulbaris gelangt aus der unteren Frontalwindung als Sprachbahn zum Hypoglossuskern. Der Tractus strio-thalamicus verbindet das Striatum mit dem Thalamus.

Zum grössten Teil aber sind diese Verbindungen nur aus klinischen und experimentellen Beobachtungen erschlossen deren Bewertung durchaus nicht verringert werden soll - und die morphologischen Beweise für eine direkte und nicht vermittelte Verbindung stehen aus. So wie die Sache bei den Nichtsäugern lag, war es nicht möglich mit Bestimmtheit zu behaupten, ob diese Bahnen sich durch das Vereinsgebiet ohne Unterbrechung in das metamere Gehirn fortsetzen oder erst durch dessen Vermittlung jener Zusammenhang erzielt wird, wie dies ja für viele andere Bahnen feststeht.

Zum Teil darum verhielt ich mich 1906 (10) skeptisch der ersten Annahme gegenüber und meine Skepsis konnte selbst durch eigene Beobachtungen am Putorius-Hirn, nach denen das kaudale Ende des basalen Vorderbirnbündels bis an die Brücke gelangt und welches Präparat ich auch abbildete (1. c. Textfig. 22) 
nicht beseitigt werden. Bei der Maus (9) und Fledermäusen (11) war es mir nämlich damals nicht gelungen den direkten Übergang des hintersten Endes von der basalen Yorderhirnbahn in das metamere Hirn zu erkennen, wie denn auch niemand dafür bisher den morphologischen Beweis erbracht hat. Man möge hierüber nur die Lehr- und Handbücher Köllikers (13), Bechterews (1) und Edingers $(4,5)$ u. a. vergleichen. Nirgends findet sich eine beweisende Abbildung dafür und selbst die Halbschemata vom Nagergehirn in diesen Werken versagen, nur die Vollschemata, insbesondere von Bechterew, stehen dafür ein. Aber so steht es auch in den Einzelabhandlungen.

Hier nun einmal Ordnung zu schaffen, war somit guter Rat teuer und als ich auf neuen Präparaten von Scyllium jene von Edinger 1888 gesehene Fortsetzung des basalen Vorderhirnbündels in das metamere Hirn neulich wiederfand, ging ich auch an die Aufsuchung derselben bei Reptilien und bei den Säugetieren. Die Amphibien mögen diesmal wegbleiben, der Reptilienbefund gilt auch für sie.

Für die Fische wählte ich abermals Scyllium und Salmo. Für ersteren habe ich die Vorderhirnbahnen, soweit sie sich auf das Zwischenhirn und das metamere Hirn erstrecken, auf ein Halbschema (Fig. 2) übersichtlich eingetragen.

Es sammelt sich das Mantelbündel (mb) aus allen drei Teilen des Vorderhirns (I, II, III), - welche Teile ich andern Orts ausführlich beschrieben babe (10) - und indem die drei Bündel dann, peripherst an dem Vorderbirn gelegen, nach ventrokaudal gerichtet nach dem $Z$ wischenhirn zu ziehen, vereinigen sie sich dort, wo sie das Zwischenhirn, das Brachium cerebri, hinter dem Velum transversum erreichen, zu einem einheitlichen Bündel. Dieses trennt sich aber alsbald in zwei Hälften, wovon die eine dorsal, an der Kante des Brachium, die andere lateral von der ersten, fest an ihr gelegen, im vorderen Teil des Zwischenhirns über den die Tela choroidea anterior lagert. Etwas vor dem Ganglion liabenulae (gh) weichen die beiden Bündel auseinander, wobei das dorsale $\left(\mathrm{mb}^{\prime}\right)$ als Vorderhirn-Habenularbahn in das Habenularganglion tritt.

Das untere Bündel, das eigentliche Mantelbündel, erreicht, wie ich schon früher geschildert und abgebildet habe (7, Fig. 28 C.), die Opticuswurzel und liegt ihr von aussen ganz fest an. Dies 
ist aus der zitierten Abbildung zu ersehen, denn in das. Halbschema konnte man es so nicht eintragen. Sofort hinter der Opticuswurzel zerfällt das Bündel in zwei Nebenbündel. Davon kreuzt das innere in der Commisura postoptica und das äussere gerät ungekreuzt in das Vereinsgebiet, wo dann beide enden. Dies war bisher bekannt, unbekannt blieb es aber bisher, dass etwas vor der obengenannten Teilung in zwei Unterbündel das Mantelbündel ein Nebenbündel (b) in das Tectum opticum abgibt. Es tritt somit durch diese Vorderhirn-Tektalbahn das Vorderhirn mit dem metameren Hirn in Verbindung.

Das basale Vorderhirnbündel sammelt seine Fasern sowohl aus der medianen Wand der Vorderhirnhemisphären (10, Fig. 9), als auch aus dem striatalen und dem ganzen dorsolateralen Teil derselben (1. c. Textfig. 2, bvhb) und das so an der Basis einheitlich gewordene Bündel (l. c. Fig. 10) liegt nun basalwärts in dem vorderen Teil des Zwischenbirns, im Bindearm. Es liegt unterhalb der eigentlichen Rindenlage, indessen der mit ihm parallel binziehende Funiculus olfactorio-corticalis (Fig. 2 f. oei) über jener Rindenlage in der Plexiformschichte der Rinde lagert. Das basale Vorderhirnbündel gibt keine Fasern an die Commissura anterior (ca) ab. Diese hat selbstandige Bündelsysteme.

In der angegebenen bekannten Lage erreicht das basale Torderhirnbündel das Chiasma opticum, um, soweit es nicht über und median von demselben liegt (vgl. 7, Fig. 74), auch durch seine Bündelsysteme durchzudringen. Damit ist dann die Bahn bis kaudal von dem Chiasma geraten, wo es nun am Vereinsgebiet (Fig. 2 vg) in viele Einzelbündel auseinander stiebt. Diese vielen Einzelbündelchen gelangen dann in das Vereinsgebiet, dem sog. Hypothalamus, und enden dort, und nur ein stärkeres Bündel macht davon eine Ausnabme. Es liegt dieses an der medianen

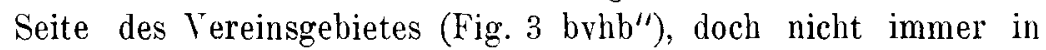
gleicher Höhe, manchmal auch ventraler (Fig. 1 bvhb"), und hier auch nicht immer als kompaktes Bündel, sondern erst noch aus mehreren Bündeln bestehend, somit diffus. Hinter dem Vereinsgebiet aber, an der Mündung des Saccus vasculosus (Fig. 4 sv), ist es stets ein kompaktes Bündel (bvhb") und lagert basalwärts und über jener Mündung. In dieser Weise erreicht es die Oculomotoriuswurzel (Fig. 1, 5 om) und liegt ihr basalwärts von der äusseren Seite an $\left(b v h b^{\prime \prime}\right)$, es kann aber sein medianer Teil von 
der Oculomotoriuswurzel auch durchzogen sein, was wohl die Regel ist. Kompakt bleibt diese Bahn bis in die Gegend des Trochlearis (Figg. 2, 6 bvbb" $^{\prime \prime}$, wo sie in basomedianer Lage über dem Ganglion interpedunculare (g. ip) lagert. Mit der Kreuzung bierselbst (Fig. 1, 6 k) hört aber diese Bahn als kompaktes Bündel auf, viele ihrer Fasern werden nach erfolgter Kreuzung zu Fibrae arcuatae, ohne dass man entscheiden könnte, dass alle ihre Fasern auf diese Weise sich verhalten würden. Tatsache ist bloss, dass diese Bahn als geschlossenes Bündel hinter der Trochlearisgegend nicht mehr besteht, was aber durchaus die Möglichkeit nicht ausschliesst, dass diffus gelagerte Fasern von ihr ungekreuzt nicht weiter kaudalwärts zögen.

Ich will nicht unterlassen, darauf hinzuweisen, dass diese Bahn in ibrem kaudalsten Verlauf schon öfter auf Querschnitten gesehen und von Edinger (4) als Tractus bulbo-thalamicus bezeichnet wurde, in der Oculomotoriusgegend aber für ihn als Tractus cerebello-thalamicus gilt (vgl. 4, Fig. 91, 138). Als beweisend aber dafür, dass $\mathrm{Edinger}$ sich hierin geirrt, ist doch immerhin die Abbildung des sagittalen Längsschnittes (von mir auf Fig. 1), aus dem deutlich die Natur dieser Bahn, als Vorderhirnbahn, hervorgeht.

Hier möchte ich noch hinzufügen, dass ich diesbezüglich das gleiche Verhalten auch bei Salmo gefunden habe. Auf Fig. 9, 10, 11, 12 habe ich diese Bahn im I. Teil meines Hirnwerkes (7) mit. vsb bezeichnet und als die gekreuzte ventrale Assoziationsbahn des Tectum opticum genannt. Dies möchte ich jetzt dahin verbessern, dass letztere Bahn jenem Bündel aus dem Vorderhirn auswärts in diffuser Form anlagert, wie auch bei Scyllium (Fig. 5 vsb).

Für die Reptilien benutzte ich meine alten Präparate von Emys und neue von grossen Exemplaren dieser Schildkröte.

Auf einem mediosagittalen Längsschnitte, der den Thalamus opticus in seiner Mitte traf, aber nach auswärts von dem Habenularganglien hinzieht und welcher Schnitt (Fig. 7) zwischen den im zweiten Teil meines Hirnwerkes (8) abgebildeten zwei Schnitten (Fig. 6 und 7) liegt, sehe ich die Verbindung zwischen Vorderhirn und der Pyramidenkreuzung (p) so dentlich, dass ich mich wundern muss, wie dieses Bündelsystem (bvhb") mir früher entgehen konnte. Es ist ja wohl wahr, dass an kleinen Exemplaren, wie das mein früheres Material war, der Zusammenhang in der 
Thalamusgegend lange nicht so deutlich ist, da das ganze Bündel von hier an nach frontalwärts zu weniger tief geschwarzt erscheint auf Markscheidenfärbungen, allein dies entschuldigt doch nicht.

Es durchzieht dieses Bündel mit den übrigen Fasern des basalen Vorderhirnbündels (bvhb) das ganze Striatum und ist dann frontal bis an die Rinde zu verfolgen. Ich verweise diesbezüglich auf einen Sagittalschnitt im zweiten Teil meiner Hirnschicht auf Fig. 2. Es ist dies Bündel hier jenes, das ich auf jener Abbildung mit $r$ bezeichnet habe und welches als Fasciculus corticalis anterior (f. sc. a) in die Stirnrinde hineinzieht. Ich sagte darüber: „Es zieht nämlich aus der Markrinde des Cortex, aus der Grenze zwischen Pallium und der Area parolfactoria, den Sulcus coronalis und die Ganglienzellschicht des Gycus coronalis durchsetzend, ein ansehnliches markhaltiges Bündel ventralwärts und versenkt sich zwischen die Fasern jener Bündel im Striatum" (l. c. S. 331). Es lässt sich dann dieses Bündel nach meinen letzten Befunden (Fig. 7 bvhb $^{\prime \prime}$ ) bis in die Oblongata verfolgen. Dabei möchte ich bemerken, dass der Fasciculus corticalis anterior nur ein Teil jenes Bündelsystems ist, das Unger (15) als Fasciculus tegmenti anführt, und von dem er einen Teil als markloses Bündel in die Commissura fornicata treten sah.

Innen liegt das Bündel dorsal (Fig. 7 bvhb $^{\prime \prime}$ ) in dem basalen Vorderhirnbündel (bvhb) und erreicht so das Vereinsgebiet (vg). Hier zwischen dem Thalamus (tho) und dem Vereinsgebiet (vg) gelegen, biegt es hinter dem Vereinsgebiet nach oben und beschreibt im metameren Hirne bis zum Kleinhirn einen nach dorsalwärts zu konvexen Bogen $\left(b_{v h b}\right.$ ). Dabei ist diese ganze Bahn im $\mathrm{Z}$ wischenhirn und dem metameren Hirnboden kein einheitlich kompaktes Bündel, sondern ein auseinandergelegenes Bündelsystem, das eben darum auf sagittalen Längsschnitten gut erkennbar, auf Querschnitten aber mit dem Fasersystem der sogenannten Haubenbahn (gemischtem Längsfasersystem des metameren Hirnes. mihi) so verwoben ist, dass ich es davon nicht zu unterscheiden vermag. So zieht denn dieses Bündel, nachdem es die Commissura ansulata passiert, in der Kleinhirngegend abwärts und verdichtet sich hier, um sich hinter dem Kleinhirn ganz basalwärts mit jener Bahn zu vereinigen, die ich als Pyramidenbahn (pyb) bezeichnet hatte. Letztere entspringt, wie ich gezeigt hatte, im Vereinsgebiet speziell im Lobus inferior (l. c. Fig. 9 pyb). 
Dass diese Bahn in der Commissura ansulata Kreuzungsfasern abgibt, erhellt wohl daraus, dass sie später weniger faserreich zu sein scheint.

Bezüglich der Säugetiere möchte ich mich vorerst nur an die Maus halten und gleich bemerken, dass ich das meiste, was über das basale Vorderhirnbündel gesagt werden soll, im dritten Teil meiner Hirnarbeit bereits beschrieben und abgebildet habe. Nur einen sagittalen Längsschnitt hatte ich nicht genügend gewürdigt. Es fällt dieser zwischen die beiden Schnitte auf Fig. 8 und 9 (9). Ich hatte dort über das basale Vorderhirnbündel gesagt (1. c. S. 431), dass, sobald es das Ganglion hypothalamicum laterale erreicht, teilen sich lateralwärts seine Bündel auf und umgreifen so von der ganzen lateralen Seite das Ganglion schalenförmig. Hierbei biegen die distalst gelegenen Fasern des Bündels im Ganglion nach aufwärts und vermengen sich vielfach mit den Pyramidenfasern. Nur ein Teil dieser Fasern verbleibt aber in dem Ganglion derselben Seite, der andere durchsetzt bloss das gleichseitige Ganglion, um dann durch die Commissura infundibularis hindurch in das anderseitige Ganglion zu gelangen .... Gerade dort, wo das Vorderhirnbündel das Ganglion erreicht, zweigt sich etwas medianwärts von der früheren Stelle ein starkes Bündel aus dem ventralsten Teil des Hauptbündels in höchst charakteristischer Weise, unter fast rechtem Winkel, ab, und begibt sich dorsomedianwärts .... Es entspringt aber dieses Bündel nicht im Grosshirn, sondern seine Fasern sind blosse Kollateraläste solcher Fasern, die aus Ganglienzellen des Ganglion hypothalamicum laterale entspringend, im basalen Vorderhirnbündel grosshirnwärts ziehen (Kölliker) ${ }^{4}$. Bezüglich jener Stelle, wo ich über die Vermengung der Fasern des basalen Vorderhirnbündels mit jenen der Pyramidenbahn im Ganglion hypothalamicum laterale sprach, sagte ich dann in einer Fussnote, dass ,,diese innige Aneinanderlagerung, besonders bei so gedrängten Zuständen, wie sie sich im Mäusegehirn finden, konnte es freilich leicht veranlassen, dass ein direkter Übergang von Pyramidenfasern und anderen Bahnen in die Grosshirnrinde angenommen wird." Hieran nun möchte ich anknüpfen.

Jener sagittale Schnitt, den ich oben genannt habe, und der zwischen die abgebildeten Schnitte (9, Fig. 8 und 9) des dritten Teiles meines Hirnwerkes fällt (Fig. 8, vorl. Arb.), ist so geführt 
(Textfig. 1 y, y), dass das basale Vorderhirnbündel in seiner Mitte der Länge nach durchschnitten ist und ist der zweite Schnitt der nach aussen zu dem auf der Fig. 8 des zitierten Werkes abgebildeten folgt. Jenes nach dorsalwärts hinaufziehende Bündel (Fig. 8 bvhb) aus der gemeinsamen Bahn (bvhb) ist noch da.

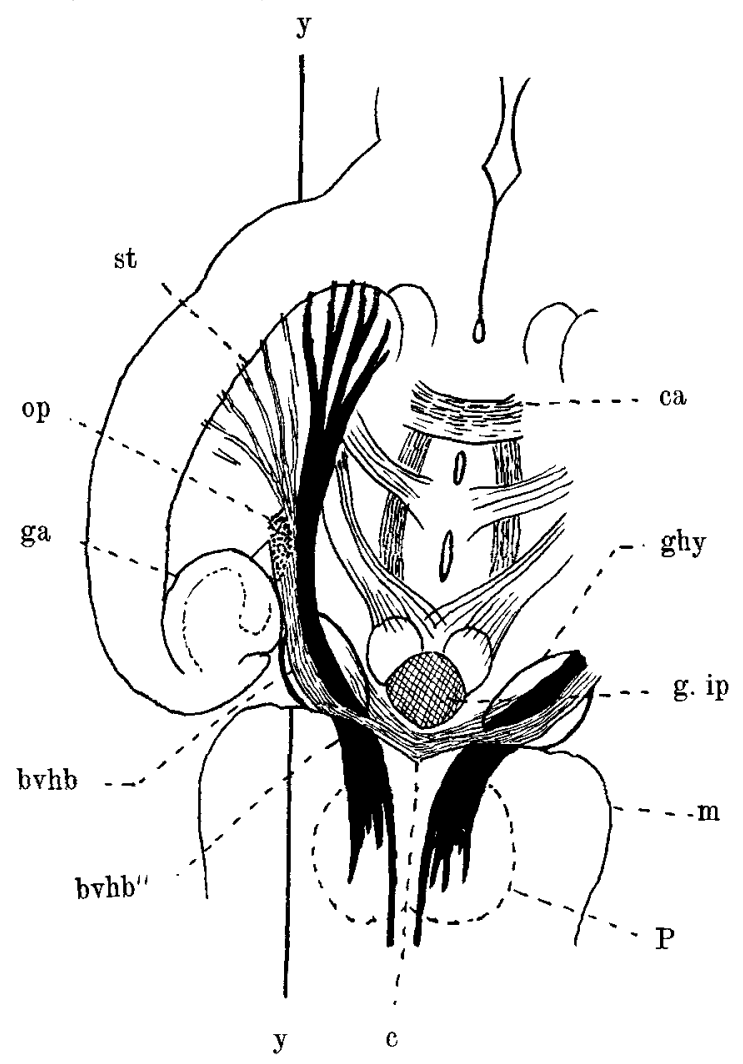

Fig. 1.

Maus. Horizontalschnitt durch das Grosshirn an dessen bodenwärtigem Teil. Die Schnittrichtung zeigt auf Fig. 8 yy. ca $=$ Commissura anterior; g. ip $=$ Ganglion interpedunculare $; \mathrm{m}=$ metameres Hirn $; \mathrm{c}=$ Commissur der basalen Vorderhirnbahn (bvhb); $\mathrm{P}=$ Pons; bvhb" $^{\prime \prime}=$ cerebrale Pyramidenbahn; ga $=$ Gyrus ammonis; st $=$ Striatum; op $=$ Opticus.

Die ganze Bahn tritt in das Ganglion hypothalamicum laterale (ghy') ein, ohne dasselbe, wie weiter auswärts, korbförmig zu umgreifen.

Allein nur in der dorsalen Hälfte dieser Eintrittsstelle lassen sich die Fasern in das Ganglion verfolgen; es sind dies jene 
Bündel, die, wie wir oben sahen und wie Kölliker uns belehrt hatte, als Zellfortsätze von Ganglienzellen aus dem Ganglion hypothalamicum laterale stirnwärts $\mathrm{zu}$ ziehen und nach dorsalwärts zu ihre Kollateralfasern zu einem Bündel (bvhb') zusammentreten lassen. Cnter dem Ganglion hypotbalamicum laterale zieht aber das Bündel (bvhb") hinweg, macht dann kaudalwärts vom Ganglion, entsprechend der Krümmung des Hirnbodens, hier gleichfalls eine nach dorsalwärts konvexe Krümmung und gelangt damit aus dem Zwischenhirn in das metamere Hirn. Hier stets bodenwärts und somit peripher gelegen, hat es gleich die Brücke (P) erreicht. Ein guter Teil seiner Fasern versenkt sich in die Brücke, der bodenwärtigste aber, gering an der Zahl, zieht unter der Brücke weiter kaudalwärts zu.

Oberhalb der Knickungsstelle des Hirnbodens vor der Brücke liegt hier über dem beschriebenen Bündel jener Teil der Pyramidenbahn (pyb), welcher etwas mehr medianwärts in voller Mächtigkeit (9. Fig. $8 \mathrm{pvb}^{\prime}$ ) in das Ganglion hypothalamicum laterale einbiegt und wie ich für die Maus, Igel und Chiropteren, dann für die Reptilien gezeigt hatte, in jenem Ganglion endigt oder beginnt. Oberhalb dieser Stelle zieht die gekreuzte laterale Assoziationsbahn der Vierhügel (amb) nach oben zu. Diese Bahn wird gekreuzt durch die sogenannte Haubenbahn (M).

Diese Bahn verliert sich im Vereinsgebiete etwas von oben nach unten zu biegend. Hier in gleicher Biegung nach kaudalwärts zu liegt vor ihr jenes Bündel des basalen Vorderhirnbündels $\left(b_{v h} b^{\prime}\right)$, welches als Kollateraläste aus dem Ganglion liypothalamicum laterale durch Kölliker erkannt ward.

Ich habe nun nach dem Befund bei Fischen und Reptilien gedacht, vielleicht hängen hier $(x)$ diese Bahnen zusammen und suchte nach einer solchen Verbindung, allein vergebens. Es splittern sich hier beide Enden der Bündel völlig auf, indem sie vorher schon marklos geworden sind.

Kaudalwärts von der Brücke sieht man die Pyramidenbahn aus nebeneinander verlaufenden Faserbündeln bestehen (pyb), die aber, wie Querschnitte zeigen (siehe 9, Fig. 30, 33-38 pyb) medianst ein dichtes Hauptbündel bilden, was aber für uns das Wichtigste ist, besteht in der Tatsache, dass in der Pyramidenbahn der Säugetiere hauptsächlich zwei Bündelsysteme nebeneinander verlaufen, von denen das eine, im 
Ganglion hypothalamicum laterale, das andere in der Grosshirnrinde sein vorderes Endgebiet bat.

Wir wollen von den verschiedenen Kaudalendgebieten der Pyramidenbahn, gekreuzten und ungekreuzten Fasern und dem Verhalten in der Brücke einstweilen absehen und nur das Ende in der Grosshirnrinde etwas näher betrachten. Dabei glaube ich mich doch keinem abermaligen Tadel auszusetzen, wenn ich die beiden Hauptbündelsysteme in der Pyramidenbahn mit verschiedenen Namen, je nach ihrem frontalen Endgebiet, bezeichne. Es sind die hypothalamale und die cerebrale Pyramidenbahn. Auf horizontalen Längsschnitten erkennt man, wie ich dies auf Fig. 27 des dritten Teiles meines Hirnwerkes abgebildet habe, das basale Vorderhirnbündel aus zwei Teilen bestehen, aus einem ausseren und einem inneren, diesem fest anliegenden. Textfig. 1 stellt hier eine vereinfachte Kopie dieser Abbildung dar, wobei das innere Bündel, das nur als cerebrale Pyramidenbahn erkannt wurde, schwarz gezeichnet (brhb") und schematisch in die Brücke (P) verlängert wurde. Was aussen von dieser Bahn vom basalen Vorderhirnbündel liegt, ist indessen jener Teil, der teilweise im gleichseitigen Ganglion hypothalamicum laterale (ghy) endet oder durch die Commissur (c) in das jenseitige Ganglion gerät. Von diesem Bündelteil können wir mit Sicherheit heute aussagen, dass seine Fasern doppelleitend sind, insofern ein Teil im Ganglion hypothalamicum laterale aus Ganglienzellen entsteht und in der Grosshirnrinde oder im Striatum aufsplittert, der andere aber in Grosshirnrinden- und Striatumzellen entsteht und im Ganglion hypothalamicum laterale aufsplittert. Hierauf hatte schon Kölliker hingewiesen (13). Das Striatum zum Teil wenigstens als ein Abkömmling der Grosshirnrinde (Fische, Reptilien, Vögel) aufzufassen, darf aber nicht in starken Gegensatz zur Rinde gestellt werden. Der Bündelteil also kann nur den Namen Tr. cerebrohypothalamicus führen.

Es bezieht die Grosshirnpyramidenbahn den grössten Teil ihrer Fasern bei den Nagern noch aus dem Stirnhirn (Textfigur), allein ein geringerer 'Teil gerät auch hier, wie Sagittalschnitte zeigen (Fig. 8), aus temporalen und vielleicht auch occipitalen Teilen des Grosshirnmantels in das Bündel. Letztere Fasersysteme erfahren dann eine ungemein grössere Vermehrung mit der zunehmenden Vergrösserung des Grosshirn- 
mantels bei Putorius, wie ich dies gezeigt habe $(9$, besonders Textfig. 18), und erlangen ihre grösste Vermehrung bei dem Menschen. Als ein frontales (Arnold sches) Bündel und kaudales ('T ürcksches) Bündel die Tractus cortico-pontini umfassen sie im Hirnschenkel den Tractus cerebro-hypothalamicus. Das A rnoldsche Bündel fasst bei dem Menschen als Weiterdifferenzierung, die Sprachbahn in sich. Doch setzt man noch eine weitere Differenzierung jenes Bündels voraus, den $\mathrm{Tr}$. cortico-spinalis. Dieser nur soll die Brücke passieren.

Wie wir nun in vorliegender Schrift sahen, ist jene einheitliche cerebrale Pyramidenbahn schon bei den Fischen aufgetreten und erhält sich in gleicher Weise bei den Reptilien. Sie hat hier scheinbar eine andere Lage wie bei den Säugetieren, eine höbere, und erreicht die basalwandige Lage erst im kaudaleren Verlauf. Dieses Verbalten andert sich mit den Säugetieren, indem das cerebrale Bündel der Pyramidenbahn eine basalwärtigere Lage gewinnt, was wohl mit der völligen Differenzierung eines Thalamus und des Vereinsgebietes erzielt wurde. Denn erst jetzt von den Reptilien an kann die Bezeichnung Hypothalamus mit der Differenzierung eines Thalamus aus dem Vereinsgebiet eine Geltung haben, da bei den Fischen mit der völligen Entfaltung eines Lobus opticus kein Thalamus bestand. Dies hatte ich bewiesen (7). ${ }^{1}$ )

Mit der starken Vorwärtsschiebung, verursacht durch Konzentration, war wieder die massige Entfaltung des Vorderhirns bedingt, und nun gelangte nicht nur das Ganglion interpedunculare weiter frontalwärts, sondern auch die Brücke entfaltete sich aus früheren Anfängen bei den Fischen. Weit hinten liegt bei diesen jene Kreuzungsstelle (Fig. $1 \mathrm{k}$ ), die später der Brücke angehört und bis wohin einstweilen die Pyramidenbahn bei diesen niederen Chordaten verfolgt werden konnte. Es versteht sich wohl von selbst, dass die bis zur Brückenkreuzung reichende Pyramidenbahn der Fische beide Bündel in sich schliesst.

Es ist die Pyramidenbahn ein gemischtes System von Längsbahnen, insofern sowohl Fasern aus Grosshirnrindenzellen als auch

1) Die Bezeichnung Hypothalamus und hypothalamal müsste konsequenterweise doch wegfallen, wenn es nicht Unvorsichtigkeit genug noch gäbe, die selbst - es ist wahr - sogar von einem Balkensystem bei Ichthyden gesprochen hätte! 
solche aus Zellen des Vereinsgebietes in sie gelangen. Es handelt sich aber jedesmal um von frontal nach kaudal, also um eine zentrifugale Leitung. Es tritt durch die Pyramidenbahn die Grosshirnrinde mit allen drei Kerngebieten (oberes, mittleres, unteres) in Beziehung. Soweit es sich aber um den Beginn im Ganglion hypothalamicum laterale handelt, dürfte es sich vielleicht auch um zentripetale Leitung nach der Grosshirninde zu handeln. Denn ich erinnere daran, dass die Grosshirnrinde mit einer grossen Zahl von metameren Hirnbahnen vermittelst des Vereinsgebietes mit der Grosshirnrinde in Beziehung steht. So z. B. mit dem Vorderstrang-Grundbündel, dessen fontales Ende im Vereinsgebiet verbleibt. In diesem Falle handelt es sich dann nur um Zuleitungen zur Grosshirnrinde und zum Striatum.

Ich schliesse somit mit der Bemerkung, dass auch die Pyramidenbahn ihren Vorläufer bei den Fischen hat, die mit der Entfaltung der Grosshirnrinde in entsprechender Weise zunimmt. Eine völlig grundlose und der Entwicklungsidee, die doch genügendlich begründet ist, widersprechende ist jene Annahme, dass eine bei den höheren Säugetieren oder Säugetieren über haupt dominierende Bahn sei ein Neuerwerb ohne Vorläufer sei.

Heidelberg, im April 1913.

\section{Literaturverzeichnis.}

1. Bechterew, v. W.: Die Leitungsbahnen im Gehirn und Rückenmark. Leipzig 1899.

2. Eding er, L.: Das Vorderhirn. Abhandl. d. Senckenbergschen naturf. Ges., Bd. XV.

3. Derselbe: Das Zwischenhirn. Ebendort, Bd. XVIII.

4. Derselbe: Vorlesungen über den Bau der nervösen Zentralorgane. Bd. II, Leipzig 1908.

5. Derselbe: Dieselben. Bd. I, Leipzig 1911.

6. Goldstein, K.: Untersuchungen über das Vorderhirn und Zwischenhirn einiger Knochenfische. Arch. f. mikr. Anat., Bd. LXVI, 1905.

7. Haller, B.: Vom Bau des Wirbeltiergehirns I. Morph. Jahrb., Bd. XXVI.

8. Derselbe: Vom Bau des Wirbeltiergehirns II. Morph. Jahrb., Bd. XXVII.

9. Derselbe: Vom Ban des Wirbeltiergehirns III, Morph. Jahrb., Bd. XXVIII.

10. Derselbe: Die phyletische Entfaltung der Grosshirnrinde. Arch. f. mikr. Anat., Bd. LXXI.

11. Derselbe: Beiträge zur Phylogenese des Grosshirns der Säugetiere. Arch. f. mikr. Anat., Bd. LXIX, 1906. 
12. Kappers, A.: The Strukture of the Teleostean and Selachian Brain. Journal of Comp. Neurologie and Physiologie, Bd. XVI, 1906.

13. Kölliker, A.: Handbuch der Gewebelehre des Menschen. 6. Aufl., Bd. II, Leipzig 1896.

14. Mayer, Fr.: Das Zentralnervensystem von Ammocoetes. Anat. Anz.. Bd. VIII, 1897.

15. Unger, L.: Das Vorderhirn des Geckos. Merkels Anat. Hefte, Bd. 31, 1906.

\section{Erklärung der Abbildungen auf Tafel XXII.}

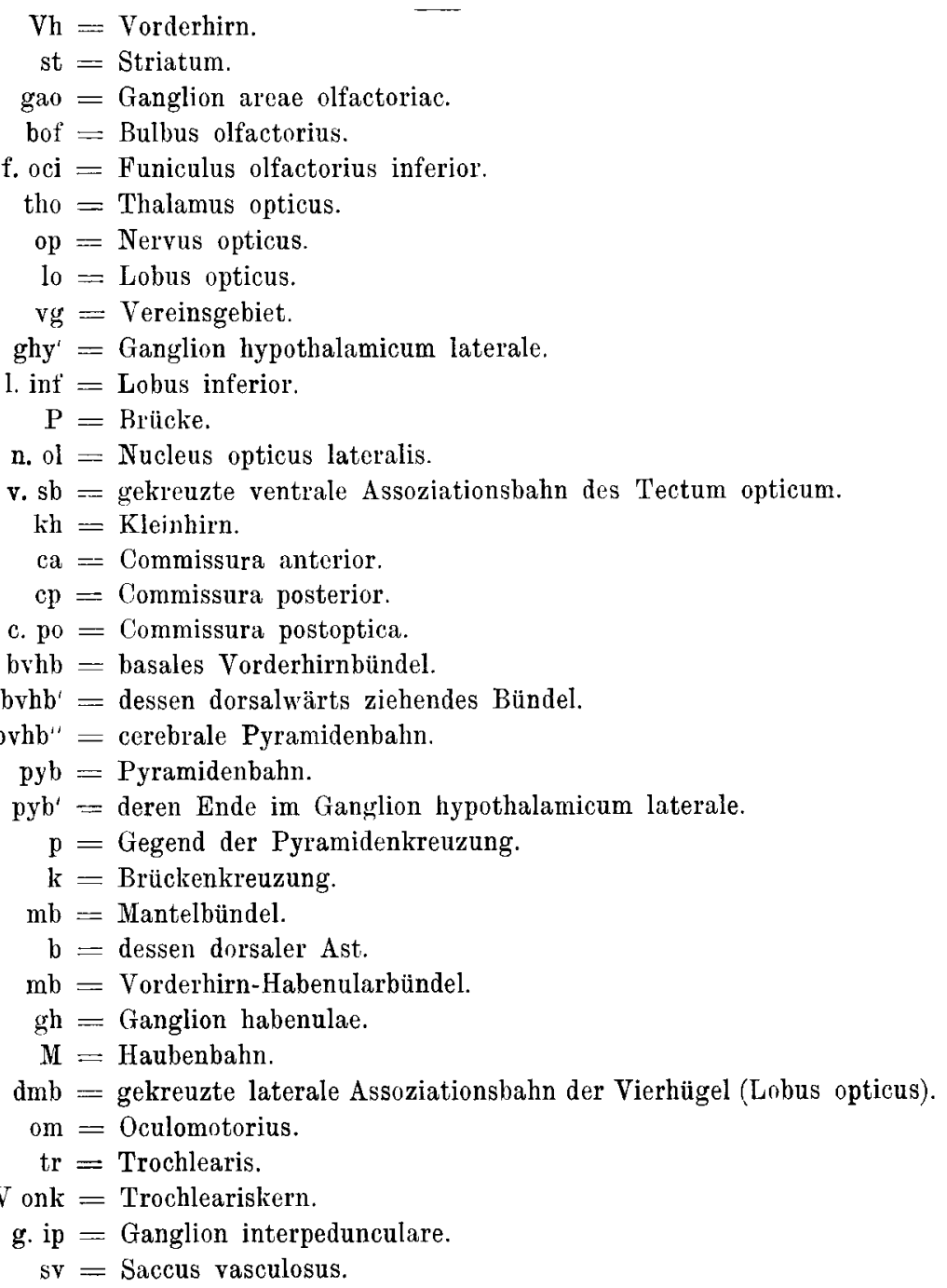


Die Verbindung des Vorderhirns mit dem metameren Hirn.

Fig. 1. Scyllium cat. Sagittaler Längsschnitt durch das Zwischen- und Mittelhirn.

Fig. 2. Scyllium. Auf einem sagitto-lateralen Längsschnitt des ganzen Gehirns sind Vorderhirnbahnen halbschematisch eingetragen.

Fig. 3. Scyllium. Querschnitt aus der Gegend der Commissura posterior.

Fig. 4. Scylli u m. Ebenso aus der Mündungsgegend des Saccus vasculosus.

Fig. 5. Scyllium. Ebenso durch die Oculomotoriusgegend.

Fig. 6. Scylli um. Ebenso durch die Trochlearisgegend.

Fig. 7. Emys. Sagitto-lateraler Längsschnitt durch das ganze Gehirn.

Fig. 8. Hausmaus. Ebenso. 